\title{
Two ABCB4 point mutations of strategic NBD-motifs do not prevent protein targeting to the plasma membrane but promote MDR3 dysfunction
}

\author{
Dario Degiorgio ${ }^{\star, 1,10}$, Paola A Corsetto ${ }^{2}$, Angela M Rizzo ${ }^{2}$, Carla Colombo ${ }^{3}$, Manuela Seia ${ }^{1}$, Lucy Costantino ${ }^{1}$, \\ Gigliola Montorfano ${ }^{2}$, Rossella Tomaiuolo ${ }^{4,5}$, Domenico Bordo ${ }^{6}$, Serena Sansanelli ${ }^{1}, \mathrm{Min} \mathrm{Li}^{7}$, Daniela Tavian ${ }^{8}$, \\ Maria P Rastaldi ${ }^{7,11}$ and Domenico A Coviello ${ }^{9,11}$
}

The $A B C B 4$ gene encodes for MDR3, a protein that translocates phosphatidylcholine from the inner to the outer leaflet of the hepatocanalicular membrane; its deficiency favors the formation of 'toxic bile'. Several forms of hepatobiliary diseases have been associated with $A B C B 4$ mutations, but the detrimental effects of most mutations on the encoded protein needs to be clarified. Among subjects with cholangiopathies who were screened for mutations in $A B C B 4$ by direct sequencing, we identified the new mutation p.(L481R) in three brothers. According to our model of tertiary structure, this mutation affects the Q-loop, whereas the p. $(\mathrm{Y} 403 \mathrm{H})$ mutation, that we already described in two other families, involves the A-loop. This study was aimed at analyzing the functional relevance of these two $A B C B 4$ mutations: MDR3 expression and lipid content in the culture supernatant were evaluated in cell lines stably transfected with the $A B C B 4$ wild-type clone and corresponding mutants. No differences of expression were observed between wild-type and mutant gene products. Instead, both mutations caused a reduction of phosphatidylcholine secretion compared with the wild-type transfected cell lines. On the contrary, cholesterol (Chol) release, after 1 and $3 \mathrm{~mm}$ sodium taurocholate stimulation, was higher in the mutant-transfected cell lines than that in the wild-type and was particularly enhanced in cells transfected with the p.Y403H-construct.In summary, our data show that both mutations do not seem to affect protein expression, but are able to reduce the efflux of phosphatidylcholine associated with increase of Chol, thereby promoting the formation of toxic bile.

European Journal of Human Genetics (2014) 22, 633-639; doi:10.1038/ejhg.2013.214; published online 18 September 2013

Keywords: MDR3; floppase activity; $A B C B 4$; lipid asymmetry; biliary cholesterol; phosphatidylcholine

\section{INTRODUCTION}

The ATP-binding cassette (ABC), subfamily B, member 4 ( $A B C B 4)$ gene $\left(\mathrm{OMIM}^{\star} 171060\right)$ encodes the MDR3 protein, a member of the $\mathrm{ABC}$ transporters superfamily, ${ }^{1}$ which is present in all major evolutionary phyla. $\mathrm{ABC}$ transporters use the energy derived from the ATP hydrolysis to provide a molecular pathway across biological membranes for the translocation of a wide variety of solutes inside ( $\mathrm{ABC}$ importer) or outside ( $\mathrm{ABC}$ exporter) the cell or its organelles. ${ }^{2}$ This common task, independent of the substrate, is driven through the activity of two structural modules, both characterized by a hydrophobic transmembrane domain (TMD) containing the substrate-binding pocket and a hydrophilic nucleotide-binding domain (NBD) suitable for ATP binding and hydrolysis. ${ }^{3,4}$ These domains can be differently organized: for example, $\mathrm{ABC}$ importers almost invariably show four individual polypeptide chains, whereas MDR3 protein is arranged as a single polypeptide chain with two TMD-NBD modules joined through a small linker region.

The classification as $\mathrm{ABC}$ transporters is inferred by the presence of eight invariant nucleotide-binding/hydrolysis motifs within the two NBDs; from $\mathrm{NH}_{2}$ to $\mathrm{COOH}$ terminal, these motifs are referred to as A-loop, Walker A motif, Q-loop region, X loop, C motif, Walker B motif, D-loop and switch motif ${ }^{3,5}$ (Supplementary Figure 1). Previously, we built the three-dimensional model of MDR3 based on the homology with the multidrug $\mathrm{ABC}$ exporter Sav1866 of Staphylococcus aureus, ${ }^{6}$ a homodimeric bacterial transporter. Both MDR3 and Sav1886 show significant homology of sequence (35\% identical residues, spanning 578 amino-acid positions) and work as exporters.

MDR3 is functionally expressed in hepatocytes, where it promotes the translocation of phosphatidylcholine (PC) across the canalicular plasma membrane, which results in the secretion

\footnotetext{
${ }^{1}$ Medical Genetics Laboratory, Fondazione IRCCS Ca' Granda Ospedale Maggiore Policlinico, Milan, Italy; ${ }^{2}$ Dipartimento di Scienze Farmacologiche e Biomolecolari, Università degli Studi di Milano, Milan, Italy; ${ }^{3}$ Department of Pediatrics, Fondazione IRCCS Ca' Granda Ospedale Maggiore Policlinico, Milan, Italy; ${ }^{4}$ Department of Molecular Medicine and Medical Biotechnologies, University of Naples Federico II, Naples, Italy; ${ }^{5} \mathrm{CEINGE}$, Advanced Biotechnologies scarl, Naples, Italy; ${ }^{6}$ RCCS Azienda Ospedaliera-Universitaria San Martino - IST. Istituto Nazionale per la Ricerca sul Cancro, Genoa, Italy; ${ }^{7}$ Renal Research Laboratory, Fondazione IRCCS Ca' Granda Ospedale Maggiore Policlinico \& Fondazione D’Amico per la Ricerca sulle Malattie Renali, Milan, Italy; ${ }^{8}$ CRIBENS-Laboratory of Cellular Biochemistry and Molecular Biology, Catholic University of the Sacred Heart, Milan, Italy; ${ }^{9}$ Human Genetics Laboratory, EO Ospedali Galliera, Genoa, Italy

10Present address: Centre for Inherited Cardiovascular Diseases, Foundation IRCCS Policlinico San Matteo, Piazzale Golgi 19, Pavia 27100, Italy.

${ }^{11}$ MPR and DAC are joint last authors.

*Correspondence: Dr D Degiorgio, Molecular Genetics Laboratory, Fondazione IRCCS Ca' Granda Ospedale Maggiore Policlinico, via S Barnaba, 8, Milan 20122, Italy. Tel: +39 025503 2433; Fax: +39 025503 2277; E-mail: dario_degiorgio@hotmail.com
}

Received 12 February 2013; revised 27 July 2013; accepted 16 August 2013; published online 18 September 2013 
of PC into the bile; $;^{7}$ more recently, it has been suggested that MDR3 activity might be indirectly correlated with biliary cholesterol (Chol) output, hence contributing to Chol homeostasis into the bile. ${ }^{8}$

The MDR3 protein is called floppase because it flops PC from the inner leaflet to the outer leaflet of the membrane, whereas other lipid translocators (i.e, ATP8B1 protein) that mediate the reverse process are known as flippases. ${ }^{9}$

Originally, two homozygous null alleles of $A B C B 4$ gene were described in two unrelated children with progressive familial intrahepatic cholestasis type 3 (PFIC-3, PFIC with elevated serum $\gamma$-glutamyl-transpeptidase $(\gamma$-GT) activity), while in one of the mothers (both heterozygous carriers of $A B C B 4$ mutation) was recorded recurrent episodes of cholestasis during the pregnancy. ${ }^{10}$ In the following years, a growing number of $A B C B 4$ mutations have been identified also in adult subjects affected by juvenile cholelithiasis, ${ }^{11}$ drug-induced cholestasis ${ }^{12}$ and idiopathic cholestasis of the adulthood. ${ }^{13}$ Recently, our group highlighted that the simple heterozygous $A B C B 4$ genotype could be a discrete risk factor for a wide range of hepatobiliary diseases, including the PFIC-3 phenotype with variable disease expressivity, ${ }^{14}$ primary biliary cirrhosis and primary sclerosing cholangitis (Crosignani $\mathrm{A}$, personal communication). To date, 146 disease-associated $A B C B 4$ mutations are reported in the Human Genome Mutation Database (http://www.hgmd.org/; release date 14 December 2012); of them, 87 (about 60\%) are predicted to substitute a single amino acid and 30 of these (about $34 \%$ ) fall within the N-terminal NBD. However, even if several ABCB4 defects have been linked to diverse hepatobiliary diseases, the detrimental effect of most missense mutations on protein function remains mainly speculative, as predicted by in silico analysis only. Because of the invasiveness of biliary sampling, in vivo evaluation of the content of biliary PC has been performed occasionally ${ }^{13,15}$ and hence both the evidences of human diseases linked to $A B C B 4$ mutations and the related pathogenic mechanisms associated with floppase dysfunction require further understanding.

To analyze the relationship between genetic changes and direct/ indirect consequences related to MDR3 protein dysfunction, we studied two missense mutations, involving the A-loop and the Q-loop of the protein, observed in three unrelated families, in which several members are affected by hepatobiliary diseases.

\section{MATERIALS AND METHODS}

\section{Subjects}

The study involved three unrelated Italian families, each with at least two members affected by $A B C B 4$-related hepatobiliary diseases.

Family A includes 15 siblings, 7 of them diagnosed with juvenile cholelithiasis and 8 clinically silent; the father died at the age of 56 with a history of cholelithiasis evolved into biliary cirrhosis (Supplementary Figure 2). We collected the blood sample from three affected siblings and their mother: a man aged 45 and a woman aged 47 reported recurrent cholelithiasis since the age of 32 and 28, respectively, persisting even after cholecystectomy or endoscopic retrograde cholangiopancreatography; the younger sister, who presented abnormal bile duct morphology and fibrosis, has had pruritus during the first pregnancy (at 25 years of age), whereas an episode of cholangitis was documented later. The mother underwent cholecystectomy for gallstones.

Family B includes a 3-year-old child diagnosed as PFIC-3 due to compensated cirrhosis with evidence of portal hypertension. His mother suffered from intrahepatic cholestasis in the late stages of pregnancy.

Family $\mathrm{C}$ includes an asymptomatic infant aged 10 months, with high levels of $\gamma$-GT $(3.5 \times)$ and serum bile acids $(33 \mu \mathrm{M})$, and his mother affected by early complicated gallstones.
At recruitment, informed consent for molecular genetic testing was obtained from all subjects. Families B and $\mathrm{C}$ have been described previously. ${ }^{14}$

\section{Genetic analysis, DNA cloning and site-directed mutagenesis}

Genomic DNA samples were isolated from blood lymphocytes and mutation analysis was performed as previously described; 6 the NCBI transcript sequence used as reference to describe our nucleotide changes was the ABCB4 isoform A, NM_000443. To exclude that the new nucleotide change identified in family A was a nonpathogenetic polymorphism, we searched this variant in 224 chromosomes of 112 healthy Caucasians ${ }^{14}$ and in the NCBI-SNP database (http://www.ncbi.nlm.nih.gov/SNP).

The nucleotide change c.1207T $>\mathrm{C}$ that encodes the mutant protein characterized by $\mathrm{p} .(\mathrm{Y} 403 \mathrm{H})$ was previously described at homozygous state in the child of family B and at heterozygous state in the other members of family B and C. ${ }^{14}$

To evaluate the expression of MDR3 in vitro, we used two plasmids: one containing the full-length cDNA of $A B C B 4$ wild type on a pcDNA3.1/ Hygro $(+)$ backbone (pcDNA3.1/Hygro $(+) / \mathrm{ABCB} 4)$ and the second containing the $\beta$-galactosidase gene (pcDNA3.1.V5/His-lacZ). They were kindly provided by Professor K Ueda (Graduate School of Agriculture, Kyoto University, Kyoto, Japan) and Dr I Garagiola (Angelo Bianchi Bonomi Hemophilia and Thrombosis Center, Fondazione IRCCS $\mathrm{Ca}^{\prime}$ Granda, Milan, Italy), respectively.

The plasmid pcDNA3.1/Hygro $(+)$ /ABCB4 was also used to obtain clones harboring the $\mathrm{p} .(\mathrm{Y} 403 \mathrm{H})$ or the $\mathrm{p} .(\mathrm{L} 481 \mathrm{R})$ mutation. The plasmid expressing $\beta$-galactosidase was used to monitor transfection efficiency. A detailed description of the targeted mutation strategy is provided in the Supplementary Material.

\section{Bioinformatic analysis}

The three-dimensional models of the human MDR3 and of the p.Leu481Arg mutant were built by homology based on the structure of the murine multidrug resistance protein $1 \mathrm{~A}$ (gene name, Abcbla), determined with crystallographic methods (PDB: 3g5u). ${ }^{16}$ The models were built with an automated procedure with the SwissModel web server (http://www. swissmodel.expasy.org). ${ }^{17} \mathrm{~A}$ recent report $^{18}$ describing the structure of the multidrug transporter P-glycoprotein from Caenorhabditis elegans has identified the ambiguous positioning of some amino acids in the membranecrossing $\alpha$-helices in the structure model of ABCB 1A. However, this should not influence the modeling of the NBD based on the structure of the same domain of the murine protein. In consideration of the high degree of sequence similarity (75\% identical residues), the model of the native human MDR3 should be considered reliable. As the ATP-binding and ATP hydrolysis motifs are conserved across the evolutionary phyla, the putative position of the two nucleoside molecules was inferred from that observed for the ADP molecules present in the bacterial multidrug $\mathrm{ABC}$ transporter Sav1886 from S. aureus (PDB: 2hyd). ${ }^{3}$

The multiple sequence alignment of amino-acid stretches from 14 species, which includes orthologous and homologous loci of p.Y403 and p.L481 MDR3, was performed by using Clustal Omega (http://www.ebi.ac.uk/Tools/ $\mathrm{msa} /$ clustalo/).

\section{Cell culture and transfection}

On the basis of literature data ${ }^{19}$ and on cell lines available in our laboratory, first experiments were performed on intrahepatic cholangiocarcinoma (HUH28), intrahepatic hepatocarcinoma (HUH7), embryonic kidney 293 (HEK293), epithelial carcinoma (HeLa), glioblastoma-astrocytoma epitheliallike (U-87MG) and endothelial capillary (EOMA) cell lines.

After first screening, the HUH7 cell line was used as positive control, whereas the HUH28 cell line was considered suitable for transfection experiments.

Western blot and immunocytochemistry methodologies are described in the Supplementary Methods. 


\section{PC and Chol dosage}

Stably transfected HUH28 cells lines were cultured in T75 flasks with highglucose DMEM supplemented with 10\% FBS until they reached a number of about 15-20 million. After being washed twice with Hank's balanced salt solution, cells were incubated for $24 \mathrm{~h}$ in DMEM $(10 \mathrm{ml})$ with or without sodium taurocholate (NaTC) $(0.5,1,3.0 \mathrm{~mm}$; Wako Chemicals $\mathrm{GmbH}$, Neuss, Germany). Subsequently, to establish the content of PC and Chol, supernatants were collected, centrifuged $(10 \mathrm{~min}, 1000 \mathrm{~g})$ to remove cell debris and lyophilized.

Lipids were extracted with three different chloroform/methanol mixtures $(1: 1,1: 2$ and $2: 1, \mathrm{v} / \mathrm{v})$ and partitioned with water and with the theoretical upper phase (chloroform/methanol/water, 47:48:1, by volume). The organic phase was dried and then suspended in chloroform/methanol $(2: 1, \mathrm{v} / \mathrm{v})$ for the analysis of PC and Chol contents.

PC and Chol analysis was achieved with an HPLC-evaporative lightscattering detector (ELSD) system (Jasco, Tokyo, Japan) equipped with one pump, a SCL-10 Advp, a degasser module and a Rheodyne manual injector with $20 \mu \mathrm{l}$ sample loop and a column (length $250 \mathrm{~mm}$, I.D $4.6 \mathrm{~mm}$ and film thickness $5 \mu \mathrm{m}$ ) packed with silica normal-phase LiChrospher Si 60 (LiChroCART 250-4; Merck, Darmstadt, Germany).

The chromatographic separation was achieved with a linear binary gradient. ${ }^{20}$ An ELSD was used to detect and separate the phospholipid species. Cells were then collected using trypsin-EDTA, centrifuged and pellets were analyzed to evaluate protein content. ${ }^{21}$

\section{Statistical analysis}

Lipid quantitative data are presented as mean \pm SE. Five experiments were performed in triplicate. Student's unpaired $t$-test was used for comparisons between samples.

\section{RESULTS}

\section{Molecular analysis and in silico evaluation}

The sequencing analysis of $A B C B 4$ gene in the members of family $\mathrm{A}$ showed a new heterozygous mutation, c. $1442 \mathrm{~T}>\mathrm{G}$, which causes the p.(L481R) amino-acid change. This mutation is absent in 112 healthy subjects and is not reported in the NCBI-SNP database; the new nucleotide change was submitted to GenBank database (accession number KC505181) at NCBI. The p.(L481R) replaces the hydrophobic Leu side chain with a bulkier and hydrophilic Arg residue in the first NBD. The structural comparison of several monomeric NBDs, ${ }^{22}$ and the modeled tertiary structure of MDR3, showed that the residue Leu481 belongs to the core of the protein's NBD and falls in a flexible amino-acid stretch known as Q-loop region containing the phylogenetically invariant glutamine residue (Q477 in MDR3). Leu481 is completely removed from the solvent (solvent-exposed surface: $0 \AA^{2}$ ), with the side chain pointing toward the core of the N-terminal NBD domain (Figure 1a). The substitution of the hydrophobic Leu residue with the bulkier and charged Arg side chain, also considering the hydrophobic environment formed by the neighboring residues (Figure 1b), is energetically unfavorable and is likely to influence the stability of the protein structure in the vicinity of the mutated site.

The multiple sequence alignment of the NBDs of Sav1866, three bacterial Msba transporters, the human and C. elegans P-glycoproteins and further eight orthologous MDR3 proteins (Supplementary Figure 3) shows that Leu481 is an invariant residue; the same evolutionary conservation has already been observed for the aromatic residue Tyr403, which belongs to the A-loop and is mutated in Hys403 in the families B and C of this study.

Evaluation of suitable cell lines for stable expression of MDR3 Endogenous expression of MDR3 was first evaluated. Western blot analysis showed MDR3 migrating at about $140 \mathrm{KDa}$ and the
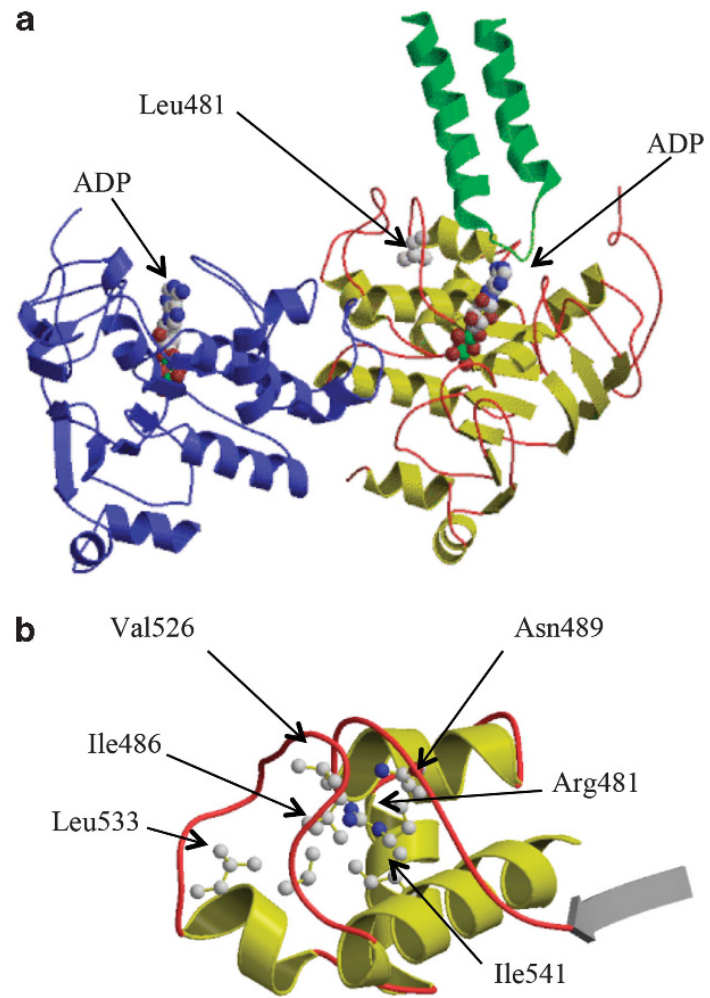

Figure 1 Ribbon representation of the tertiary structure of human MDR3. (a) The three-dimensional structure of the $\mathrm{N}$-terminal NBD (residues 375-631) built by homology with the structure of the murine multidrug resistance protein $1 \mathrm{~A}$ is represented with $\alpha$-helices and $\beta$-strands shown in yellow and the connecting loops in red. The C-terminal NBD (residues 1020-1280) is shown in blue. The mutated Leu481 and the two ADP molecules are represented in ball-and-stick. The two TMD $\alpha$-helices in the vicinity of the Leu481 (residues 884-927) are also shown in green. Carbon, oxygen, nitrogen and phosphorus atoms are colored in gray, red, blue and green, respectively. (b) Region surrounding the p.Leu481Arg mutation, with the neighboring residues represented in ball-and-stick.

immunostaining visualized the protein in the cytoplasm and on the cell surface in HUH7 and HEK293 cells, whereas the protein was less expressed or completely absent in HUH28 and EOMA.

Atypical nuclear expression was found in U-87MG and HeLa cell lines (Supplementary Figure 4); therefore, these cells were excluded from further experiments. EOMA cells were excluded as well because they were more resistant to transfection.

In $\mathrm{HUH} 28$ cells, transient transfection with pcDNA3.1/Hygro $(+) /$ ABCB4 induced the expression of an expected product of about $140 \mathrm{kDa}$, and resulted in cytoplasmic and membrane immunostaining (Figures $2 \mathrm{a}$ and $\mathrm{b}$ ).

Stably transfected HUH28 cell lines expressing human MDR3 The selected point mutations were singly introduced by site-directed mutagenesis. Mutant plasmids were sequenced and the electropherograms showed the expected nucleotide changes at positions c.1207 $(\mathrm{T}>\mathrm{C})$ and c.1442 $(\mathrm{T}>\mathrm{G})$ (Supplementary Figure 5). HUH28 cells were therefore transiently (Figure 2) and then stably (Figure 3) transfected with wild-type and mutant forms of ABCB4 construct. Both mutants were expressed at approximately the same level as the wild-type protein (Figure 3a). Experiments were extensively repeated, showing stable expression at different time points. Both MDR3 

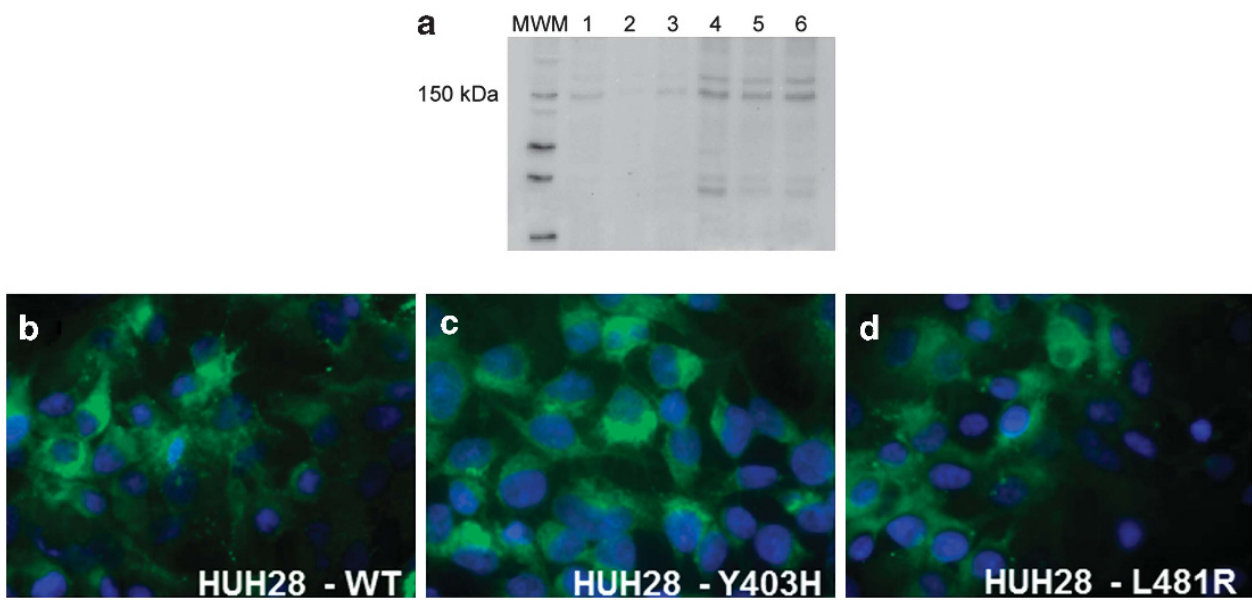

Figure 2 Expression of wild-type and mutant $A B C B 4$ after transient transfection. (a) Representative western blot results, showing that a band of about $140 \mathrm{kDa}$ is present in non-transfected HUH7 cells (lane 1, positive control) and absent from non-transfected HUH28 cells (lane 2). The same band can be observed with different degrees of intensity in HUH7 cells transiently transfected with wild-type ABCB4 at $48 \mathrm{~h}$ (lane 3 ) and $72 \mathrm{~h}$ (lane 4 ), as well as in HUH28 cells transiently transfected with wild-type ABCB4 at 48h (lane 5) and $72 \mathrm{~h}$ (lane 6). (b-d) By immunofluorescence, HUH28 cells transiently transfected with wild-type (b) or mutant (c, d) ABCB4 display similar expression and location of the protein. Indirect IF, DAPI nuclear counterstain, magnification $\times 200$.
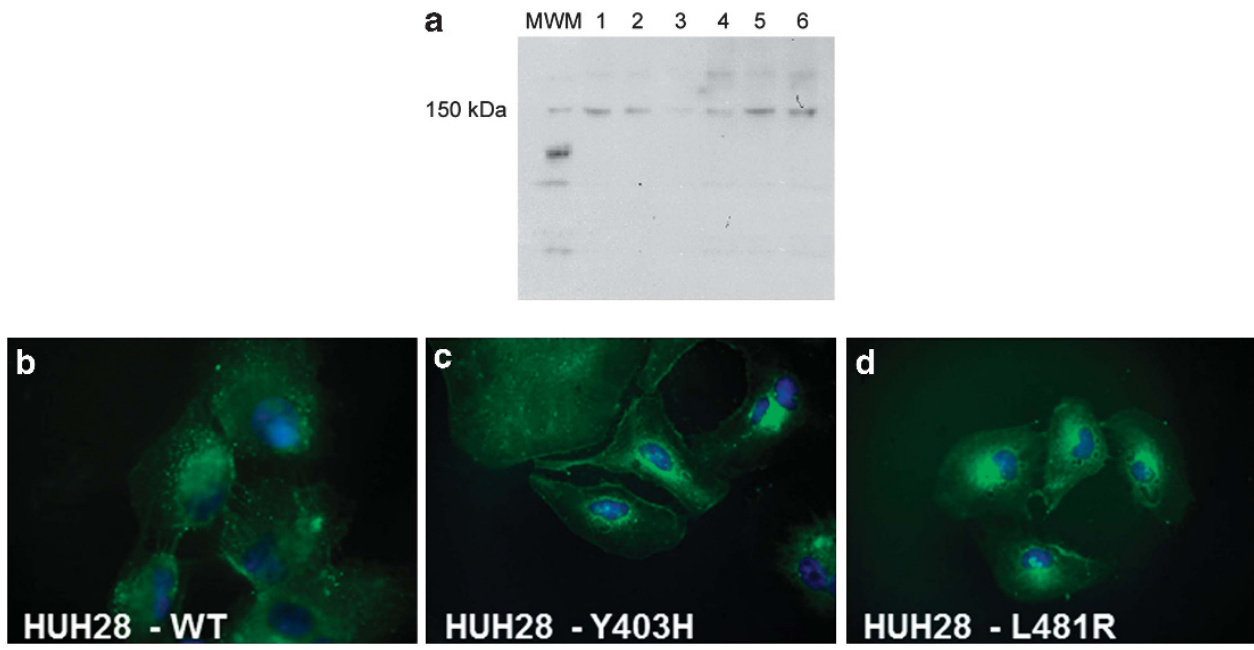

Figure 3 Expression of WT and mutant $A B C B 4$ in stably transfected cell lines. (a) Representative western blot results, showing that a band of about $140 \mathrm{kDa}$ is present in wild-type ABCB4 stably transfected HUH28 cells (lane 1) and HUH7 (lane 2), and absent/barely positive in non-transfected HUH28 cells (lanes 3 and 4). Lanes 5 and 6 show the stable expression of p.L481R-ABCB4 and p.Y403H, respectively. (b-d) By immunofluorescence, HUH28 cells stably transfected with wild-type (b) or mutant (c, d) ABCB4 display similar expression and location of the protein. Indirect IF, DAPI nuclear counterstain, magnification $\times 400$.

mutants did not display distinct subcellular location as compared with the wild-type protein (Figures $3 \mathrm{~b}-\mathrm{d}$ ).

PC and Chol determination in HUH28 cell lines expressing wildtype and mutant MDR3

PC and Chol evaluation in the supernatant was performed to compare stably transfected HUH28 cells expressing the wild-type protein and those expressing the mutants. Wild-type cells secreted more PC and Chol than the mutant ones; PC: $3.417 \mathrm{nmol} / \mathrm{mg}$ proteins versus 1.410 for p.L481R and 2.346 for p.Y403H and Chol: $4.476 \mathrm{nmol} / \mathrm{mg}$ proteins versus 3.193 for p.L481R and 3.811 for p.Y403H (Figures 4a and b; Supplementary Table 1).

Treatment with NaTC $(0.5,1$ and $3 \mathrm{~mm})$ induced a significant dosedependent increase of PC secretion in all cells (Figure 4a). Although wild-type cells and p.Y403H mutant showed similar fold increases in PC excretion, p.L481R mutant showed higher sensitivity to NaTC reaching 11-fold increase in $\mathrm{PC}$ secretion at $3 \mathrm{~mm}$ compared with the absence of NaTC (Supplementary Table 1). Nevertheless, mutant cells did not reach upon NaTC stimulation the amount of PC secreted by wild-type MDR3-transfected cells.

Stimulation with 1.0 and $3.0 \mathrm{~mm}$ NaTC increased Chol excretion in both wild-type and mutant cells (Figure 4b); in these conditions, a greater dosage of Chol was always associated with the two mutant cell lines. In particular, p.Y403H displayed higher Chol excretion compared with wild-type cells at $3.0 \mathrm{mM}$ NaTC: 9.04 versus 6.05 by referring at parameter 'fold increase' and 34.438 versus 27.074 (nmol/mg proteins) by referring at parameter 'absolute amount'. On the contrary, $0.5 \mathrm{~mm}$ NaTC did not have any effect on Chol excretion 

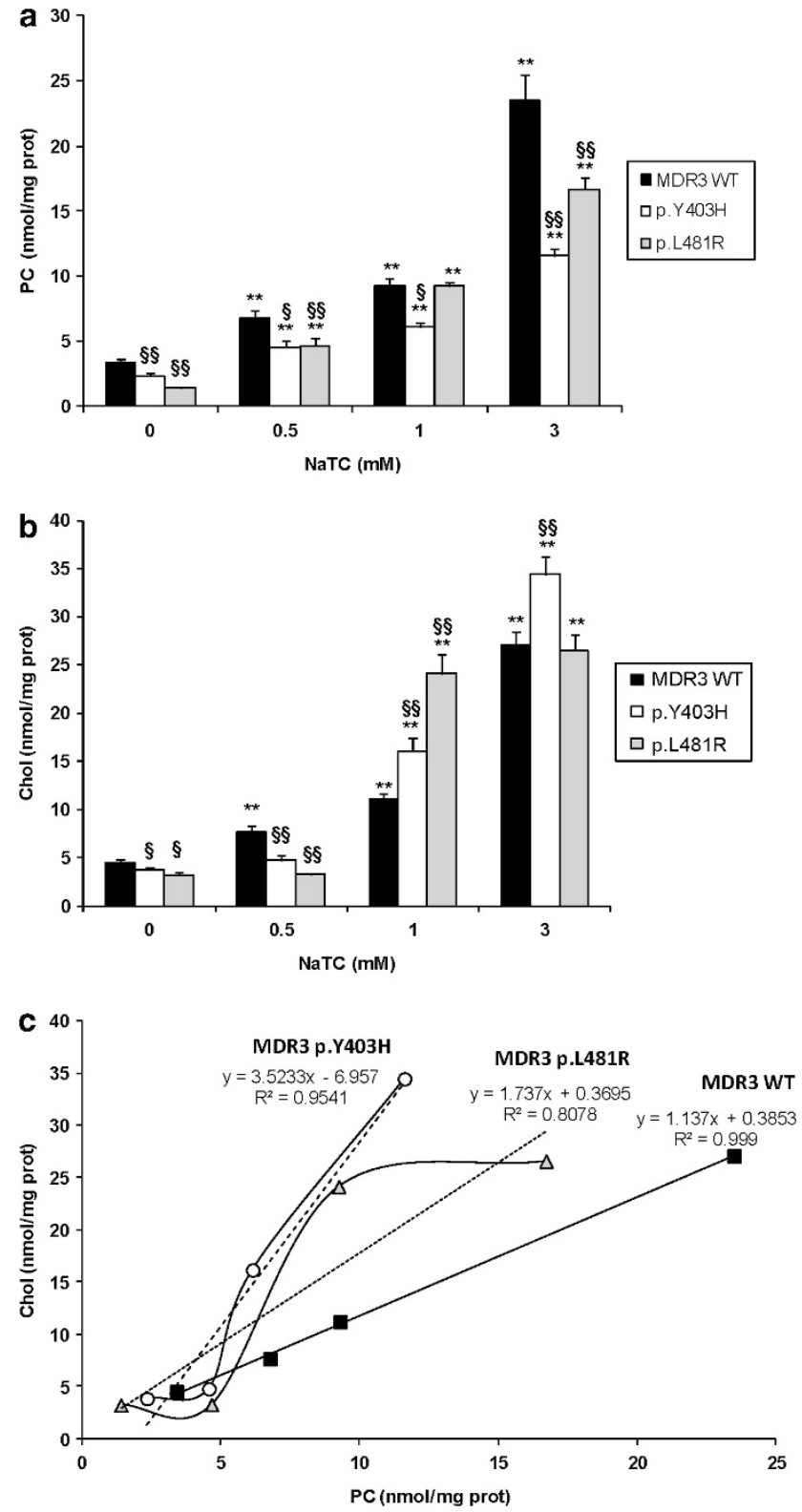

Figure $4 \mathrm{PC}$ and Chol determination. Analysis of PC (a) and Chol (b) secretion, and PC-Chol correlation (c) in MDR3 wild-type, MDR3 p.Y403H and MDR3 p.L481R cells. Cells were incubated for $24 \mathrm{~h}$ at $37^{\circ} \mathrm{C}$ in the absence $(0 \mathrm{mM})$ or presence of $0.5 \mathrm{mM}$, or $1 \mathrm{mM}$ or $3 \mathrm{mM} \mathrm{NaTC}$. The values represent the mean $\pm S E$ of five experiments performed in triplicate. Asterisks indicate values significantly different (Student's unpaired $t$-test) from control values $\left({ }^{*} P \leq 0.05\right.$ and ${ }^{* *} P \leq 0.01$ versus 0 NaTC). Symbols ( $\$$ ) indicate significant differences between mutant and wild-type cells after the same NaTC stimulus $\left({ }^{\S} P \leq 0.05\right.$ and ${ }^{\$} P \leq 0.01$ versus MDR3 WT at the same NaTC concentration).

in both mutant cell lines, but increased Chol excretion only in wildtype cells (1.7-fold increase compared with control; Supplementary Table 1).

The PC/Chol ratio showed a similar trend in absence or with 1.0 and $3.0 \mathrm{~mm}$ of NaTC; it was reduced in both mutants, particularly in $\mathrm{Y} 403 \mathrm{H}$ cells, whereas an inverted trend was shown after treatment with $0.5 \mathrm{~mm}$ NaTC.

To better evaluate these data, we plotted the correlation between PC and Chol medium contents. As reported in Figure 4c, in mutant
Y403H cells low values of PC efflux are associated with high Chol into the culture medium.

\section{DISCUSSION}

The role of the missense mutations in the pathogenesis of $A B C B 4$ related disease remains speculative because only in a few studies ${ }^{19,23,24}$ their pathogenicity has been correlated with direct or indirect evidence of anomalous floppase activity.

In this study, besides reporting the identification of a novel $A B C B 4$ mutation (p.(L481R)) within the Q-loop in three siblings suffering from juvenile cholelithiasis, we demonstrate that this mutation, stably transfected in HUH28 cells, as well as the mutation p. $(\mathrm{Y} 403 \mathrm{H})$, previously described by our group, ${ }^{14}$ do not prevent protein targeting to the plasma membrane but induce an abnormal efflux of PC and Chol.

Although MDR3 defects are rare, ${ }^{6}$ several findings suggest that MDR3-floppase dysfunction has a substantial direct and indirect influence on human cholestatic morbidity. Recently, Groen et al 25 have shown a functional interdependence between MDR3-floppase activity (that promotes outward translocation of inner leaflet PC) and ATP8B1-flippase activity (that promotes inward translocation of outer leaflet phosphatidylserine); only the concerted activities of both transporters allow the necessary balance of phospholipids through the bilayer of the hepatocanalicular membrane. Moreover, this 'lipid asymmetry', a mechanism that ensures protection of hepatocytes against the detergent action of bile salts, needs to be maintained in an active mode through MDR3 function, as the formation of nontoxic bile (i.e, of mixed bile salt micelles-PC-Chol where bile salt detergent activity is reduced) is ensured by the continuous excretion of PC into the canalicular lumen by bile salts. ${ }^{9}$

The pathogenetic potential of both mutations is suggested by segregation analysis. The p.(L481R) mutation was identified in three out of seven affected siblings belonging to family A, whereas the already characterized p. $(\mathrm{Y} 403 \mathrm{H})$ mutation $^{6,14}$ was identified in four affected members belonging to other two families. According to what is generally described, ${ }^{13-15}$ the genotypes reported here show that (i) two mutant $A B C B 4$ alleles are associated with severe early-onset of intrahepatic cholestasis characterized by a rapid course toward terminal liver failure. In particular, the infant bearing the homozygous p. $(\mathrm{Y} 403 \mathrm{H})$ mutation was diagnosed as PFIC-3 at the age of 3 months, and at the age of 3 years presented compensated cirrhosis with portal hypertension; (ii) a single mutant allele can be associated with hepatobiliary diseases with less significant and highly variable clinical features. As an example, among our patients with single heterozygous mutation, juvenile cholelitiasis was diagnosed in three cases, biochemical cholestasis in two cases and intrahepatic cholestasis of pregnancy in one. As for the latter case, simple heterozygous genotypes with $A B C B 4$ mutation are considered a genetic susceptibility factor, resulting in disease only if concomitant risk factors are associated. ${ }^{14,25}$

A substantial deficit of the floppase activity has been also predicted for both mutations because they are located within two structural motifs of the N-terminal NBD involved in ATP hydrolysis, as suggested by the homology model of MDR3 built on the crystal structure of the homologous murine multidrug resistance protein 1A. These motifs represent invariant loci from bacteria to man. The involved amino-acid substitutions are associated with two amino-acid changes very unfavorable from an energetic viewpoint.

The functional aspect has been further developed through the establishment of three stably transfected HUH28 cell lines expressing wild-type and mutant MDR3. 
By WB analysis, our data seem to show in both mutants a band of similar molecular weight as the wild-type protein, and the immunostaining did not evidence expression differences, supporting previous data from our group ${ }^{14}$ and from the literature. ${ }^{19}$ The canalicular MDR3 immunostaining was detectable in about $60 \%$ of the hepatocytes of the child with PFIC-3 carrying the homozygous amino-acid change from tyrosine to hystidine in the codon $403 .{ }^{14}$ Moreover, the mutagenesis of this aromatic residue that introduces the amino acid tryptophan in the mouse Mdr1A protein did not result in a targeting-defective protein in the plasma membrane of yeast, but caused impaired ATPase transport and greatly reduced activity. $^{26}$

As expected, compared with the wild type, the amount of PC secreted was reduced in both mutant-transfected cell lines. Moreover, when stably transfected cells were treated with NaTC, a molecule already documented as capable to stimulate the efflux of lipids by MDR $3,{ }^{19}$ the data obtained showed a lower PC efflux for both mutant-transfected cell lines and similar or higher sensitivity of mutant proteins to NaTC stimulation when compared with the wild type.

As for biliary Chol, it is widely documented that in mice it is excreted into the canalicular lumen through different pathways; among them, a relevant rate of efflux is because of the direct activity of the specific ABC hemitransporters G5 and G8 (ABCG5 and ABCG8). ${ }^{27}$

Our data show that, in absence or low concentration of NaTC, mutant cells have less Chol efflux ability than wild-type cells, and this direct proportionality between reduced excretion of biliary PC and Chol was originally described in $\mathrm{Mdr} 2^{-1}-$ mice.?

Instead, higher concentrations of NaTC seem to increase Chol efflux by mutant cells, with a maximum value obtained in presence of the p.Y403H mutation so that the ratio between PC and Chol is inverted. This phenomenon could be explained by the concept of rupture of the 'lipid asymmetry', caused by the $A B C B 4$ mutations, indicating that a severe alteration of floppase activity only, in a context where flippase activity is maintained to wild-type levels, could prevent the appropriate phospholipidic gradient across the canalicular membrane that is necessary to protect from detergent properties of toxic bile. ${ }^{25}$ The passive extraction of Chol from the outer leaflet of the plasma membrane, mediated by NaTC not complexed with an appropriates amount of PC, is hence plausible.

According to our results, comparable changes have been reported in the bile of Mdr2 wild-type and $\mathrm{KO}$ mice during intravenous infusion with increasing amounts of taurocholate: ${ }^{25,28}$ a simultaneous increase of PC and Chol was documented in the wild-type animals, whereas an inverted $\mathrm{PC} / \mathrm{Chol}$ ratio was observed in the $\mathrm{KO}$.

As already demonstrated, ${ }^{25}$ the expression of ABCB4 wild type may be detrimental to the vitality of the cell. In fact, even if both mutant cell lines show deficiency of floppase activity, curiously the relative fold increase in PC secretion, upon NaTC treatment, appears very similar or higher in the mutants compared with the wild type. In addition, the deleterious effect on wild-type MDR3 was particularly evident when the cells were treated with $0.5 \mathrm{~mm}$ NaTC: only in this condition, the $\mathrm{PC} / \mathrm{Chol}$ ratio showed a higher value in mutants with respect to wild-type cells; hence, it is plausible that, for HUH28 cell line, this low concentration of NaTC does not represent a sufficient stimulus for wild-type MDR3 compared with mutants.

In summary, we have functionally characterized two $A B C B 4$ mutations located in the A- and Q-loop within the N-terminal NBD motif of MDR3 protein that are associated with different cholangiopathies in the affected members of three families, demonstrating that both mutations can be responsible for disease.
Our data support the hypothesis that MDR3 dysfunction favors the disruption of 'plasma membrane phospholipid asymmetry' and point out the possibility of identifying patients who may benefit from a pharmacological chaperone approach to restore the defective transport activity of mutant MDR3 proteins.

\section{CONFLICT OF INTEREST}

The authors declare no conflict of interest.

\section{ACKNOWLEDGEMENTS}

We thank the patients who agreed to participate in this study. We thank those who generously provided the cell lines: Drs F Colombo and L Porretti (HUH28 and HUH7 cell lines), Dr L Torielli (HEK293 cell line), Dr F Costa and Prof N Gagliano N (U-87MG cell line). We thank Dr E Benzoni for valuable technical assistance; and Professor Castaldo G, Drs L Castellano and A Ragozzino for clinical advice. This work was supported by the grant 'PROGETTI A CONCORSO 2009-2010' of 'Fondazione IRCCS Ca Granda' (DD) and two grants of Regione Lombardia, DG Sanita' no. 12298 (2004) (CC), DG Sanita' no.19081 (2005) (CC). Part of the results from this study were presented at the 2012 European Human Genetics Conference, 23-26 June, 2012, Nürnberg, Germany.

1 Nicolaou $M$, Andress EJ, Zolnerciks JK, Dixon PH, Williamson C, Linton KJ: Canalicular ABC transporters and liver disease. J Pathol 2012; 226: 300-315.

2 Davidson AL, Chen J: ATP-binding cassette transporters in bacteria. Annu Rev Biochem 2004; 73: 241-268.

3 Dawson RJ, Locher KP: Structure of a bacterial multidrug ABC transporter. Nature 2006; 443: 180-185.

4 Rees DC, Johnson E, Lewinson O: ABC Transporters: the power to change. Nat Rev Mol Cell Biol 2009; 10: 218-227.

5 Hollenstein K, Dawson RJ, Locher KP: Structure and mechanism of ABC transporter proteins. Curr Opin Struct Biol 2007; 17: 412-418.

6 Degiorgio D, Colombo C, Seia $\mathrm{M}$ et al: Molecular characterization and structural implications of 25 new ABCB4 mutations in progressive familial intrahepatic cholestasis type 3 (PFIC3). Eur J Hum Genet 2007; 15: 1230-1238.

7 Smit JJ, Schinkel AH, Oude Elferink RP et al: Homozygous disruption of the murine mdr2 P-glycoprotein gene leads to a complete absence of phospholipid from bile and to liver disease. Cell 1993; 75: 451-462.

8 Oude Elferink RP, Beuers U: Targeting the ABCB4 gene to control cholesterol homeostasis. Expert Opin Ther Targets 2011; 15: 1173-1182.

9 Oude Elferink RP, Paulusma CC: Function and pathophysiological importance of ABCB4 (MDR3 P-glycoprotein). Pflugers Arch 2007; 453: 601-610.

10 de Vree JM, Jacquemin E, Sturm $\mathrm{E}$ et al: Mutations in the MDR3 gene cause progressive familial intrahepatic cholestasis. Proc Natl Acad Sci USA 1998; 95 282-287.

11 Rosmorduc O, Hermelin B, Boelle PY, Parc R, Taboury J, Poupon R: ABCB4 gene mutation-associated cholelithiasis in adults. Gastroenterology 2003; 125 452-459.

12 Lang C, Meier Y, Stieger B et al: Mutations and polymorphisms in the bile salt export pump and the multidrug resistance protein 3 associated with drug-induced liver injury. Pharmacogenet Genomics 2007; 17: 47-60.

13 Gotthardt D, Runz H, Keitel V et al: A mutation in the canalicular phospholipid transporter gene, $\mathrm{ABCB} 4$, is associated with cholestasis, ductopenia, and cirrhosis in adults. Hepatology 2008; 48: 1157-1166.

14 Colombo C, Vajro P, Degiorgio D et al: Clinical features and genotype-phenotype correlations in children with progressive familial intrahepatic cholestasis type 3 related to ABCB4 mutations. J Pediatr Gastroenterol Nutr 2011; 52: 73-83.

15 Jacquemin E, De Vree JML, Cresteil D et al: The wide spectrum of multidrug resistance 3 deficiency: from neonatal cholestasis to cirrhosis of adulthood. Gastroenterology 2001; 120: 1448-1458.

16 Aller SG, Yu J, Ward A et al: Structure of P-glycoprotein reveals a molecular basis for poly-specific drug binding. Science 2009; 323: 1718-1722.

17 Arnold K, Bordoli L, Kopp J, Schwede T: The SWISS-MODEL Workspace: a web-based environment for protein structure homology modelling. Bioinformatics 2006; 22: 195-201.

18 Jin MS, Oldham ML, Zhang Q, Chen J: Crystal structure of the multidrug transporter P-glycoprotein from Caenorhabditis elegans. Nature 2012; 490: 566-569.

19 Morita SY, Kobayashi A, Takanezawa Y et al: Bile salt-dependent efflux of cellular phospholipids mediated by ATP binding cassette protein B4. Hepatology 2007; 46: 188-199.

20 Corsetto PA, Montorfano G, Zava S et al: Effects of n-3 PUFAs on breast cance cells through their incorporation in plasma membrane. Lipids Health Dis 2011; 10: 73 . 
21 Lowry OH, Rosebrough NJ, Farr AL, Randall RJ: Protein measurement with the Folin phenol reagent. J Biol Chem 1951; 193: 265-275.

22 Jones PM, George AM: Mechanism of ABC transporters: a molecular dynamics simulation of a well characterized nucleotide binding subunit. Proc Natl Acad Sci USA 2002; 99: 12639-12644.

23 Dixon $\mathrm{PH}$, Weerasekera N, Linton KJ et al: Heterozygous MDR3 missense mutation associated with intrahepatic cholestasis of pregnancy: evidence for a defect in protein trafficking. Hum Mol Genet 2000; 9: 1209-1217.

24 Delaunay JL, Durand-Schneider AM, Delautier D et al: A missense mutation in ABCB4 gene involved in progressive familial intrahepatic cholestasis type 3 leads to a folding defect that can be rescued by low temperature. Hepatology 2009; 49: 1218-1227.
25 Groen A, Romero MR, Kunne $\mathrm{C}$ et al: Complementary functions of the flippase ATP8B1 and the floppase ABCB4 in maintaining canalicular membrane integrity. Gastroenterology 2011; 141: 1927-1937.

26 Carrier I, Urbatsch IL, Senior AE, Gros P: Mutational analysis of conserved aromatic residues in the A-loop of the $A B C$ transporter ABCB1A (mouse Mdr3). FEBS Lett 2007; 581: 301-308.

27 Voloshyna I, Reiss $A B$ : The $A B C$ transporters in lipid flux and atherosclerosis. Prog Lipid Res 2011; 50: 213-214.

28 Oude Elferink RP, Ottenhoff R, van Wijland M, Frijters CM, van Nieuwkerk C, Groen AK: Uncoupling of biliary phospholipid and cholesterol secretion in mice with reduced expression of mdr2 P-glycoprotein. J Lipid Res 1996; 37: 1065-1075.

Supplementary Information accompanies this paper on European Journal of Human Genetics website (http://www.nature.com/ejhg) 